\title{
ESTUDIO DEL FLUJO DE AIRE Y LA RESISTENCIA NASAL EN NIÑOS CON EDADES COMPRENDIDAS ENTRE LOS SEIS Y LOS DOCE AÑOS
}

Christian Contreras Peláez ${ }^{1}$

Palabras claves: Respiración, flujo de aire respirado, neumotacografia, resistencia nasal

\section{RESUMEN}

Dada la incertidumbre existente con respecto a la posible relación entre los desórdenes respiratorios y las alteraciones del crecimiento y desarrollo del macizo craneofacial, se pretende con la presente investigación aportar al conocimiento científico datos claves acerca de la elaboración, calibración y validación de instrumentos confiables para la medición del flujo, la resistencia nasal y las caídas asociadas de presión del aire inspirado y/o espirado, en aras de poder cuantificar los valores de normalidad de la función respiratoria.

Para tal efecto y en una primera fase del proyecto de investigación, se diseñaron, construyeron y calibraron los instrumentos de medición de la función respiratoria. Mediante el uso de un espirómetro y un neumotacógrafo, junto con transductores de presión conectados a un poligrafo, se estudiaron el flujo de aire y la resistencia nasal, el área equivalente de sección transversa nasal y el tipo oral o nasal de respiración.

Como resultado de lo anterior, se obtuvieron curvas de calibración tanto para el flujo de aire como para la diferencia de presión, presentando éstas unos coeficientes de correlación (r) de $0.9997 \mathrm{y}$ 0.9992 , respectivamente.

Este aporte logístico significa un paso dado con miras en la siguiente fase del proyecto de investigación, la cual consiste en la realización de un estudio piloto.

\section{INTRODUCCIÓN}

Actualmente, aún es significante la prevalencia de desórdenes respiratorios. Múltiples factores, intrínsecos y extrínsecos, juegan un papel trascendental dentro de su etiopatogenia. Una multicausalidad que contempla aspectos genéticos y ambientales, ha sido propuesta como la explicación de todas estas alteraciones.

El presente estudio, pretende crear en nuestro medio una infraestructura tanto locativa como humana, con el propósito de incursionar en el área investigativa de la fisiología respiratoria. Lo anterior implica la realización de un estudio piloto que servirá tanto para la calibración en la obtención de los datos, como para su aplicación clínica. En la primera fase, se construyeron y calibraron los instrumentos de medición, tanto del flujo de aire y de la resistencia respiratoria, como de los cam-

'Odontólogo Odontopediatra. Profesor Escuela de Odontologia, Universidad del Valle. 
bios de presión en el tracto respiratorio alto. Además, se requirieron instrumentos de medición confiables como el poligrafo, el espirómetro, el neuomotacógrafo y los transductores de presión.

La literatura reporta un escaso número de estudios realizados en la población infantil en los que se aborde la temática de la influencia de los transtornos respiratorios sobre el crecimiento y desarrollo craneomaxilar, por lo que sería de gran interés para nuestro medio el llevar a cabo un estudio mediante el cual se pueda evaluar y cuantificar variables que conciernen a la normal función de la ventilación en un grupo de edad preadolescente. El proyecto de investigación representa una necesidad sentida por especialistas del área otorrinolaringológica, ortopédica maxilar y quirúrgica, dada la carencia de instrumentos de medición confiables del flujo y resistencia nasal en el medio universitario.

\section{OBJETIVO GENERAL}

Analizar la función respiratoria infantil mediante la utilización de los debidos instrumentos de medición y determinar los valores normales correspondientes.

\section{OBJETIVOS ESPECÍFICOS}

Construir un instrumento de medición del flujo de aire y de la resistencia nasal de fácil manipulación, confortable para el sujeto infantil, de sencilla lectura y alta reproducibilidad.

Medir la diferencia de presión al paso del aire en la via aérea superior.

Elaborar y/o obtener un equipo de medición que cuantifique el volumen de respiración nasal y oral simultáneamente.

\section{REVISIÓN DE LA LITERATURA}

En un estudio experimental realizado por Vig et al ' en veintiocho adolescentes y adultos, utilizando un neumotacógrafo como instrumento de medición, concluyeron que el uso de una máscara para medir los patrones respiratorios de reposo, exige un minucioso control, ya que puede inducir a resultados indeseables. Se ha establecido que la resistencia nasal en el momento de la inspiración, es similar a la del momento de la espiración (Berkinshaw et $\mathrm{al}^{2}$ ). Con el fin de estandarizar el método, se sugirió utilizar un bajo flujo de aire inspirado $(0.25 \mathrm{l} / \mathrm{s})$, el cual sería fisiológico en personas jóvenes. Warren et $\mathrm{al}^{3}$, utilizaron un modelo mecánico simple de la vía aérea superior y estudiaron experimentalmente la dinámica del movimiento de aire bajo situaciones controladas. Posteriormente, Warren ${ }^{4}$ en un estudio piloto realizado en dieciocho adultos y veintiséis niños, estimó cuantitativamente las dimensiones de la vía aérea nasal, analizando así la función respiratoria normal y alterada. Los resultados sugirieron que el uso de transductores de presión asi como la medición del área de sección transversa nasal, permitian cuantificar la función respiratoria. Según Hinton et $\mathrm{l}^{5}$, la manera subjetiva como se observa la dificultad respiratoria nasal, es tal vez, la responsable de las diferencias de los valores de flujo $\mathrm{y}$ resistencia observadas por los investigadores. Ellingsen $\mathrm{et}^{\mathrm{a}} \mathrm{l}^{6}$, concluyeron que la colocación de máscaras nasales podía inducir una predisposición, al sugerir una ruta especifica para el flujo de aire. Lund', estimó que la rinomanometría posterior presentaba una apreciable desventaja: alrededor del $25 \%$ de los pacientes reportaban una importante dificultad en relajar el paladar blando, aparte de que algunos no resistían la tentación de succionar el catéter. Hamilton y Christman ${ }^{8}$, reportaron un instrumento computadorizado, apto para medir la resistencia de la vía aérea nasal. Warren et $\mathrm{al}^{9}$, utilizaron en un estudio experimental una muestra de 102 niños libres de congestión nasal, en edades comprendidas entre los 6 y los 15 años; mediante la utilización de un pletismógrafo respiratorio inductivo, midieron la respiración oronasal de reposo y mediante el uso del neumotacógrafo, hallaron el área de sección transversa nasal. Encontraronque ésta última, se incrementó aproximadamente $0.032 \mathrm{~cm}^{2}$ por año entre los 6 y 14 años de edad. 


\section{MATERIALES Y MÉTODOS}

Este proyecto es la primera fase de un estudio piloto a realizarse a posteriori. Comprende la construcción de un equipo de trabajo idóneo que permite cuantificar el flujo de aire y la resistencia nasal, como también, determinar la partición de la ventilación entre las vías nasal y oral. Este estudio se enmarca dentro de un contexto de desarrollo tecnológico.

En la cuantificación de la resistencia nasal se utilizó un polígrafo Grass modelo 7P1, el cual es un amplificador de corriente continua DC con alta sensibilidad y estabilidad. Además, se implementó un neumotacógrafo de Fleisch, que mide la caida de presión al paso del aire a través de estructuras cuasicapilares, gracias a la conexión a un transductor de presiones diferenciales volumétricas PT-5-A Grass Instrument Co. y a una bomba rotodinámica Warren E. Collins Inc., impulsada por un motor Dayton 2M066 AC DC, 115 voltios, $1 / 15 \mathrm{HP}, 5000 \mathrm{rpm}$, al que se le controló su voltaje de entrada utilizando un transformador variable Variac. Se contabilizó por medio de un cronómetro eléctrico Lab-Chron 1400 timer, 115 voltios, 60 ciclos, el tiempo que demoraba la campana del espirómetro en adquirir un volumen determinado para unas condiciones de voltaje dadas, lo que permitía hacer el cálculo del flujo de aire en litros/segundo. De igual manera, se implementó una máscara de buceo TUSA a la cual se le retiró su válvula de drenaje, adaptándole en vez, una manguera corrugada de $1 / 2$ " de diámetro, centrándose ésta de tal forma que fuera sujeta tanto por los dos tornillos que originalmente aseguraban la válvula de drenaje, como por silicona transparente. A esta máscara se le adaptó una válvula construída en PVC siguiendo el modelo de la válvula de Douglas.

Para la prueba de repartición de gases, además de la máscara, se utilizó una boquilla de caucho comúnmente requerida en pacientes sometidos a procedimientos con anestesia general y se le adaptó una manguera corrugada de $1 / 2$ " de diámetro. Para asegurar la boquilla en cada uno de los pacientes se requirió adherir una correa ajustable tipo velcro de $100 \mathrm{~cm}$ de longitud. De igual forma, se implementó tanto el uso del espirómetro de campana de Tissot, el cual sirvió como fuente reservoria de aire, como el de las dos llaves de Douglas, las cuales permitieron el cambio rápido de la dirección del flujo de aire. A estas últimas iban conectadas las bolsas colectoras de aire.

\section{CALIBRACIÓN DE EQUIPOS}

En el presente estudio se tomaron medidas de volúmenes de aire almacenados en el espirómetro (medidas estáticas) y medidas de flujo en volumen de aire/ tiempo (medidas dinámicas).

Mediciones con el Espirómetro:

El espirómetro de Tissot o de campana es un instrumento de medición balanceado en donde se verificó que en condiciones de reposo la presión interior no difiere de la del ambiente en más de $1 \mathrm{~mm}$ de $\mathrm{H} 2 \mathrm{O}$, lo que corresponde a la mayor precisión obtenible en las lecturas. Así mismo, no hubo diferencias de presión legibles asociadas con lo flujos máximos ensayados, por lo que se consideró que en todas las condiciones del presente proyecto, la presión interior del espirómetro era prácticamente igual a la atmosférica.

Los volúmenes almacenados en el espirómetro se midieron sobre el margen de la polea del aparato y para permitir la aproximación de 0.1 litro, se contó con el auxilio de un Vernier. En todos los casos se verificó y ajustó en caso necesario, la corrección del cero de la escala (ver Figura 1).

Las mediciones de flujo se hicieron introduciendo o retirando aire del interior del espirómetro con la ayuda de una bomba para gases Dayton 2M066 AC DC, 115 voltios, 1/15 HP, 5000 rpm, conectada a la salida de un autotransformador Variac, cuyo voltaje determinó la magnitud del flujo. Una vez ajustado el Variac para un flujo cercano al deseado, se iniciaba el desplazamiento del aire en un momento dado, siguiendo visualmente los valores del contenido de aire en el espirómetro y 
cuando el o los medidores de presión asociados indicaban un régimen estable, se medía el tiempo asociado con el paso de 30 litros de aire. Las medidas de presión asociadas a flujos se hicieron empleando un manómetro de agua en forma de " $U$ ", de ramas verticales iguales provistas de una escala en milimetros, leyendo los cambios de altura del menisco en una de las ramas. Se usó agua adicionada de detergente para disminuir la magnitud del menisco.

\section{Calibración del Neumotacógrafo de Fleisch:}

El aparato se dejaba aproximadamente media hora con la corriente de calefacción aplicada y durante ese lapso se balanceaba el puente medidor, usando en el polígrafo la(s) amplificación(es) utilizada(s) durante las observaciones en pacientes. Debido a que fue necesario utilizar los escalones de amplificación más altos, el registro resultaba ruidoso, por lo que se empleó sistemáticamente la amortiguación de $12 \mathrm{~Hz}$, que suprimió la mayor parte del ruido y prácticamente no afectó el registro de flujo respiratorio.

La calibración consistió en determinar las diferencias de presión entre el comienzo y el fin de la lámina arrollada en espiral, asociadas con los diversos flujos del rango de interés. Las presiones se midieron en este caso mediante un transductor diferencial PT-5-A Grass Instrument Co., y se registraron en el canal número 1 del polígrafo bajo la forma de desviaciones o deflexiones con relación a una línea de base situada cerca del centro del canal. Para la calibración se añadió un manómetro de agua conectado a las dos vías que unen el neumotacógrafo con el transductor, sobre el cual podian leerse directamente las diferencias de presión. La entrada del neumotacógrafo se unió mediante un tubo de media pulgada con la bomba Dayton manejada a través de un Variac y conectada por su otro extremo al espirómetro. En una fase inicial se registró el cero del registro, imponiendo un flujo bajo y actuando sobre las llaves de tres vías disponibles para tal fin. Para cada medida se estableció el flujo de la magnitud de- seada (juzgada por la diferencia de presión), y una vez estabilizado el registro se cuantificó el flujo midiendo tiempos y volúmenes como se indicó a propósito del espirómetro. Se hicieron registros cubriendo el rango de $9 \mathrm{~cm}$ de $\mathrm{H} 2 \mathrm{O}$, con escalones de $1 \mathrm{~cm}$ y con flujos en las dos direcciones (ver figura 2). El poligrafo y sus componentes asociados con el neumotacógrafo no se cambiaron en el curso de este trabajo y se usaron siempre con los mismos ajustes. Todos los registros tienen señales similares de calibración del puente que por limitaciones del equipo se hicieron con una amplificación mucho menor, siendo sensiblemente iguales en sesiones sucesivas.

Calibración del Sistema Medidor de Diferencias dePresión Nasofaringe-Ambiente:

Debido a las limitaciones del equipo se emplearon dos transductores de presión P23G de Statham, para medir separadamente la presiones en los sitios mencionados; sus señales alimentaron a un "Transducer Mixer" de Grass, cuya salida (igual a la diferencia de presiones) se introdujo en otro canal del polígrafo con el cual estaba conectado el neumotacógrafo. La calibración consistió en hacer fluir el aire mediante una pequeña bomba a través de una "T" de vidrio conectada con un segmento de caucho comprimible de manera gradual con una prensa de Hoffmann, mientras se medía con un manómetro de agua las diferencias de presión surgidas entre la entrada a la " $T$ " y el final del segmento compresible. Variando el ajuste de la prensa era posible obtener las diferencias de presión de $1,2,3, \ldots$, hasta $9 \mathrm{~cm}$ de $\mathrm{H} 2 \mathrm{O}$. Operando sobre un sistema de vías cruzadas se pudo invertir la dirección del flujo en el aparato, para así obtener la otra rama de la curva. Con antelación se balanceó el sistema en el "Transducer Mixer", primero en un transductor, luego en el otro y finalmente la combinación de éstos en el canal poligráfico (ver Figura 2).

Como en el caso anterior, fue necesario utilizar amplificaciones grandes resultando en ruído, por lo que se empleó una amortiguación que redujo a 
la mitad la amplitud a una frecuencia de $16 \mathrm{~Hz}$. Se registró un señal de calibración a una amplificación de $0.05 \mathrm{mV} / \mathrm{cm}$.

Medición de los Registros Poligráficos:

Las deflexiones se leyeron con buena iluminación y mediante una lupa de $7 \mathrm{X}$ provista de una escala con divisiones de $0.1 \mathrm{~mm}$ (Bausch \& Lomb Measuring Magnifier). De esta forma, era posible aproximar a ojo y en forma reproducible $0.02 \mathrm{~mm}$.

\section{RESULTADOS}

Se calculó la curva de regresión lineal mediante el programa gráfico Sigma Plot versión 4 , hallando que las pruebas de calibración del polígrafo en sus canales 1 (flujo de aire) y 2 (diferencias de presión), describieron unas gráficas cuyas curvas presentaron coeficientes de correlación ( $\mathrm{r}$ ) de 0.9997 y 0.9992 , respectivamente.

Puesto que en la primera etapa del trabajo sólo se hicieron mediciones de resistencia en espiración, se empleó únicamente la correspondiente rama de la curva de calibración, siendo una recta con los parámetros $\mathrm{Ao}_{0}=0.00771$ y $\mathrm{Al}=0.04765$ (ver figura 3 ).

La curva de calibración correspondiente a las diferencias de presión es llamativa por su angulación, probablemente atribuible al "Transducer Mixer". De ella, se obtuvieron los parámetros $\mathrm{Ao}=0.3756$ y $\mathrm{Al}=0.3572$ (ver figura 4 ).

\section{DISCUSIÓN}

La mayoría de las investigaciones realizadas en este campo han validado la metodología aquí descrita para medir la resistencia nasal tomando como premisa la interrelación entre el flujo de aire y las diferencias de presión (Berkinshaw et $\mathrm{al}^{2}$, Warren et $\mathrm{al}^{3}$, Hamilton y Christman?). Particularmente, cuando esta resistencia fue medida conociendo un flujo de aire estándar, no se suscitaron errores provenientes de todo el armazón rino-orofaringeo. $\mathrm{El}$ uso de este equipo de trabajo programado a ratas de flujo de aire estandarizadas podria ayudar a determinar el flujo óptimo de aire, el cual seria usado con el objeto de estudiar los cambios en la resistencia nasal, además de establecer directas comparaciones con los datos reportados por la literatura.

Este estudio es el primero de una serie de investigaciones cuyo propósito es el de definir en términos objetivos, la ventilación en la vía aérea superior.

\section{SUMMARY}

There is a lack of studies that give relevant evidence of the possible relation between respiratory problems and impairment of craniofacial development. This study reports about the manufacture, calibration and validation of instruments that measure breathing flow, nasal resistance and pressure of inhaled/expelled air in order to quantify the values of normal respiratory function.

A device was prepared using an espirometer and a pneumotacograph integrated to traducers connected to a polygraph to evaluate the air flow, the nasal resistence, the equivalent area of transverse nasal section and the type of breathing (oral or nasal). There were obtained calibration curves to air flow and to pressure difference, with correlation coefficients (r) of 0.9997 and 0.9992 , respectively.

\section{REFERENCIAS}

1 Vig. P. S., Sarver, D. M., Hall, D. J., Warren, D. W.: Quantitative evaluation of nasal airflow in relation to facial morphology. Am J Orthod 79 (3): 263-272. 1981.

2 Berkinshaw, E. R., Spalding, P. M., Vig, P. S.: The effect of methodology on the determination of nasal resistance. Am J Orthod Dentofac Orthop 92:329335. 1987.

3 Warren, D. W., Lehman, M. D., Hinton, V.A.: Analysis of simulated upper airway breathing. Am J Orthod 86 (3): 197-206. 1984.

4 Warren, D. W:A quantitative technique for assessing nasal airway impaiment. Am J Orthod 86(4): 306312. 1984. 
5 Hinton, V. A., Warren, D. W., Hairfield, W. M., Seaton, D.: The relationship between nasal crosssectional area and nasal air volume in normal and nasally impared adults. Am J Orthod Dentofac Orthop 92(4): 294-298. 1987.

6 Ellingsen, R., Vandevanter, C., Shapiro, P. Shapiro, $\mathrm{G}$ : Temporal variation in nasal and oral breathing in children. Am J Orthod Dentofac Orthop 107: 411417. 1995.

Figura 1.

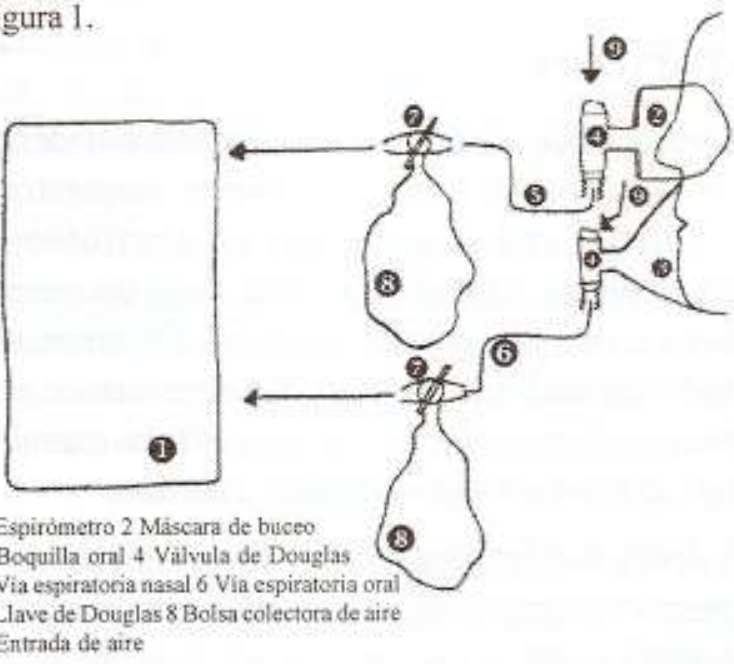

Figura 3

Calibracion del Sistema Neumotacogrifices

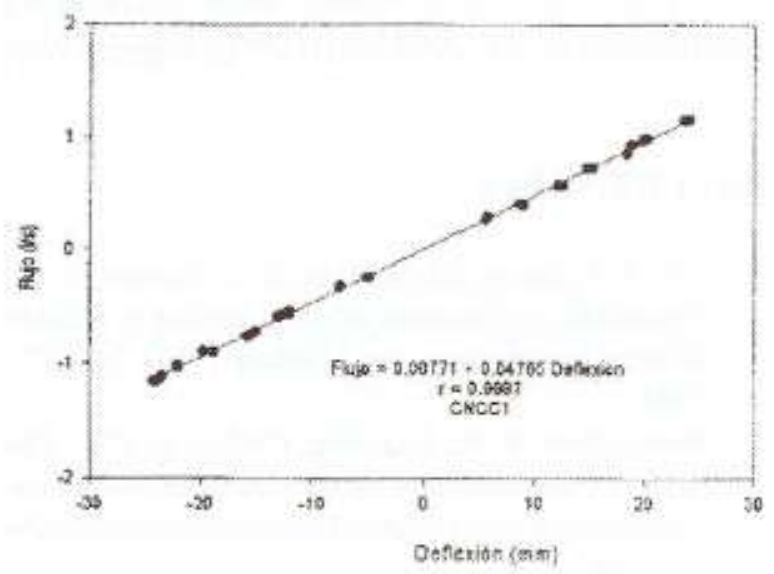

7 Lund, V. J.: Objective assesment of nasal obstruction. Otolaryngologic Clinics of North America 22(2): 279-290, 1989.

8 Warren, D. W., Hairfield, W. M., Dalston, E. T.: Effect of age on nasal cross-sectional area and respiratory mode in children. Laryngoscope 100:89-93.1990.

9 Hamilton, L. H., Christman, N. T.: Nasal airway resistance computer. The Laryngoscope 87 (11): $1945-$ 50.1977 .

Figura 2.

Prueba de flujo y de diferencia de presión.

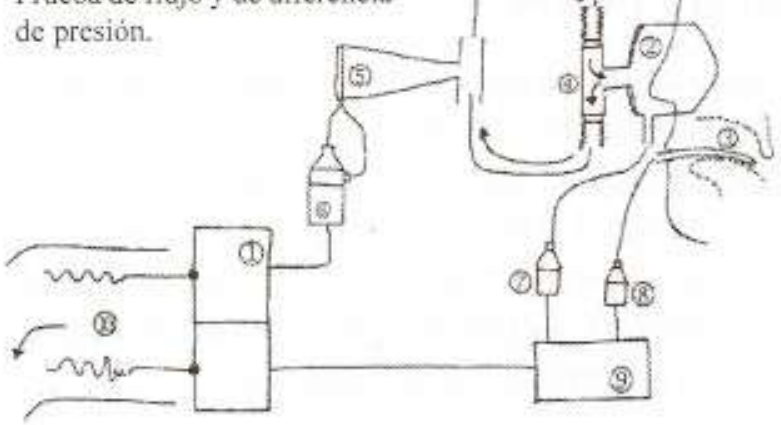

1 Poligrafo 2 Máscara de buceo

3 Cateter oral 4 Válvula de Douglas

5 Neumotacógrafo 6 Iransducter diferencial de presión

7 Transductor de presión metálice 8 Transductor de presion plästico

9 Transductor nixer 10 Papel de registro

Figura 4

Calibración del Sistema Medidor de Diferencias de Presión DP

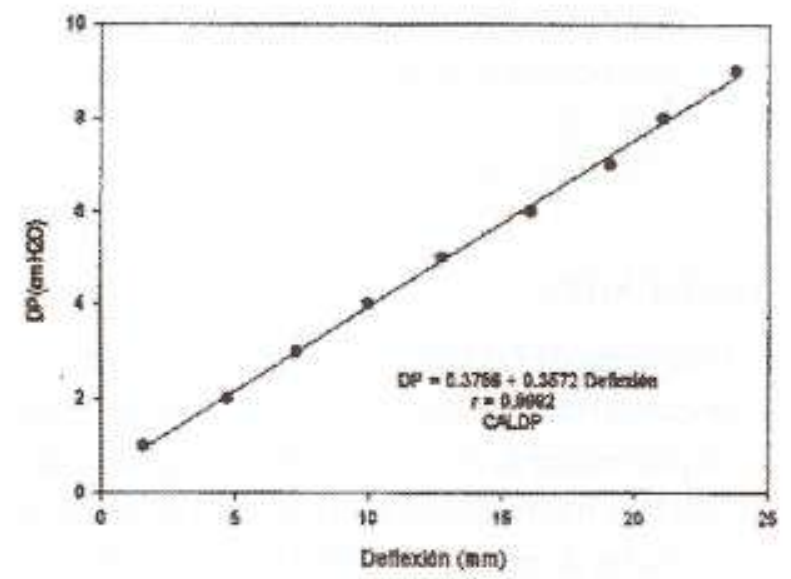

Correspondencia:

Christian Contreras Peláez

escuadon@univalle.edu.co'

Universidad del Valle

Cali 